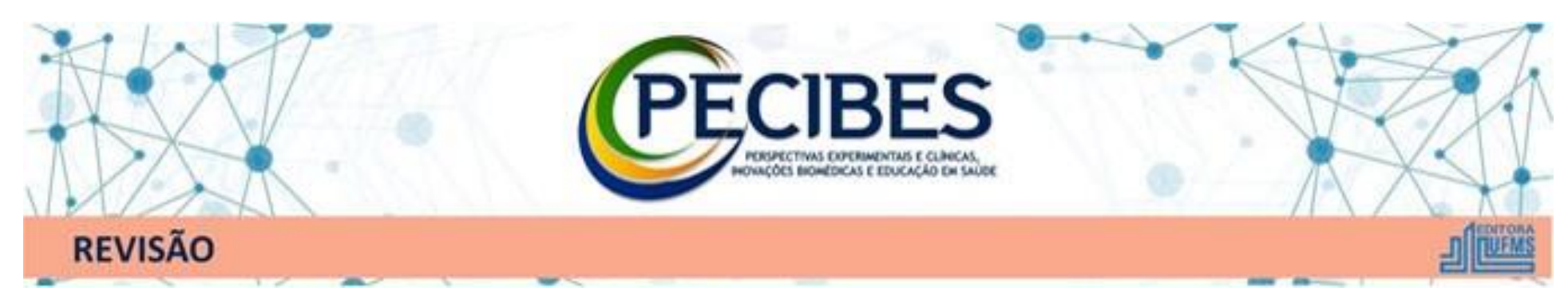

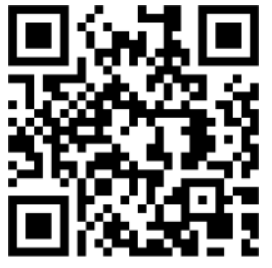

http://www.seer.ufms.br/index.php/p ecibes/index

*Autor correspondente: Willyan Franco Hilario

E-mail do autor:

willyan.hilario@ebserh.gov.br

Palavras-chave: Câncer.

Leucemia Linfocítica

Aguda. Linfoma. Leucemia

Linfoblástica.

Key-words: Cancer. Acute

Lymphoid Leukemia.

Lymphoma. Lymphobastic

Lymphoma.

\section{Principais alterações hematológicas da Leucemia Linfocítica Aguda} (LLA)

Main hematological changes in Acute Lymphocytic Leukemia (ALL)

Willyan Franco Hilario ${ }^{1}$, Lívia Silveira de Moraes Hilario ${ }^{2}$

1 Farmacêtico da Empresa Brasileira de Serviços Hospitalares (EBSERH) lotado no Hospital Universitário Maria Aparecida Pedrossian (HUMAP), Mestre em Bioquímica e Farmacologia pela Universidade Federal do Espírito Santo (UFES)

2 Farmacêutica-Bioquímica da Empresa Brasileira de Serviços Hospitalares (EBSERH) lotada no Hospital Universitário Maria Aparecida Pedrossian (HUMAP), Mestre em Bioquímica e Farmacologia pela Universidade Federal do Espírito Santo (UFES)

Resumo

A leucemia é um tipo de câncer caracterizado pela proliferação exacerbada de células leucocitárias imaturas oriundas da medula óssea. A leucemia linfocítica aguda é o tipo mais comum de câncer em crianças de 2 a 10 anos de idade. Com prevalência de $70 \%$ para estas e apenas $30 \%$ em adultos tal enfermidade é caracteriza pela rápida evolução e pela presença maciça de linfoblastos na medula óssea, sangue periférico e em outros órgãos. O quadro anêmico mais observado é normocítico normocrômico acompanhado de trombocitopenia. A classificação morfológica da LLA em L1, L2 e L3 é dirigida por características clínicas e por aspectos citomorfológicos e citoquímicos da medula e sangue periférico do paciente. O objetivo deste artigo é descrever, pontuar e revisar as principais alterações hematológicas e a classificação da LLA no contexto de um laboratório de hematologia clínica de rotina.

\section{Abstract}

Leukemia is the denomination of cancer characterized by exacerbated proliferation of immature leukocytes from the bone marrow. Acute lymphoid leukemia (ALL) is the most common type of cancer in children between 2 and 10 years old. In children its incidence is $70 \%$ while in adults is $20 \%$ only. The quick evolution and the massive number of lymphoblast in the bone marrow, peripheral blood and others organs have been charactering this disease. Additionally, normochromic normocytic anemia and thombocytopenia is frequently found in pacients with ALL. Its morphological classification in L1, L2 and L3 is driven by pacient clinical condition and citomorphologicals and citochemicals features of bone marrow and peripheral blood. In this context, our study aimed to describe and point out the mains hematological changes and the ALL classification in a routine clinical hematologic laboratory 


\section{Introdução}

Segundo o Instituto Nacional de Câncer (INCA) as leucemias representam cerca de 5,2\% dos casos de câncer no Brasil, apesar de representar um dos menores percentuais entre as neoplasias de relevância clínica, o número de casos vem crescendo anualmente (BRASIL, 2018). Entre as leucemias, a leucemia linfocítica (LLA) aguda é mais comum em crianças de 2 a 10 anos de idade, tendo prevalência de $70 \%$ para estas e apenas $30 \%$ em adultos (Pezzini e Castro, 2014). A presença de células leucocitárias imaturas, linfoblastos, no sangue periférico em decorrência da proliferação celular exacerbada, é uma das características marcantes da doença que contribui para seu diagnóstico (Fadel, 2010). Por se tratar de uma enfermidade de início subido e rápida evolução, seu reconhecimento e diagnostico precoce é de importância vital para estabelecer o prognostico clínico e dirigir a melhor conduta terapêutica (Coccaro et al., 2019).

Nesse contexto, o laboratório de hematologia por meio da análise pormenorizada dos os elementos do sangue periférico (hemograma) e de análises citoquímicas e imunohematológicas, seja no âmbito hospitalar ou ambulatorial, tem um papel crucial na detecção, identificação e diagnóstico das alterações hematológicas relacionadas à LLA (Callera, 2005).

O objetivo deste artigo de revisão é descrever e pontuar as principais alterações hematológicas e a classificação da LLA no contexto de um laboratório de hematologia clínica de rotina. Adicionalmente, o trabalho visa enriquecer e contribuir para o aperfeiçoamento de profissionais, residentes e graduandos que atuam ou possam a vir a atuar diretamente nesse campo, além de ser uma fonte de pesquisa imediata e consisa sobre o tema.

\section{Material e Métodos}

A pesquisa bibliográfica foi realizada nas bases de dados Medline, Scielo e PubMed de estudos clínicos, básicos e de revisão publicados em revistas com reconhecido impacto no meio acadêmico e científico. A pesquisa restringiu-se a trabalhos publicados com a busca de palavras-chaves: "Câncer", "Leucemia Linfocítica Aguda", "Linfloma" e "Leucemia lonfoblástica".

\section{Resultados}

As leucemias são caracterizadas pela proliferação exacerbada de células leucocitárias imaturas oriundas da medula óssea, que com a evolução da doença atingem frequentemente o sangue periférico e outros órgãos (Pezinni e Castro, 2014). Considerando a linhagem e o grau de maturação celular as leucemias podem ser subdivididas em mieloides e linfoides e classificadas em agudas e crônicas (FARIAS; CASTRO, 2004; Pezzini e Castro, 2014). Portanto, tendo em vista isso, as leucemias podem ser classificadas em Leucemia Mieloide Aguda (LMA), Leucemias Mieloide Crônica (LMC), Leucemia Linfocítica Aguda (LLA) e Leucemia Linfocítica Crônica (LLC) (Fadel, 2010).

A LLA é uma neoplasia maligna de rápida evolução decorrente da proliferação de células linfoides imaturas ou indiferenciadas (linfoblastos) presentes na medula óssea, timo e gânglios linfáticos (Farias e Castro, 2004; Pezzini e Castro, 2014). Nestes locais, pode-se observar grande infiltração dos linfoblastos em diferentes estágios de maturação, contudo, permanecem indiferenciados (Farias e Castro, 2004). Embora a incidência da LLA não seja restrita a idade, raça e sexo ela é mais comum em crianças menores de 15 anos, brancas e do sexo masculino (Fadel, 2010; Pezzini e Castro, 2014). Com prevalência de 70\% para crianças de 2 a 5 anos e apenas $30 \%$ em adultos as causas que desencadeiam a LLA são pouco esclarecidas, porém, a exposição a radiação, a certos vírus, a produtos químicos e fatores genéticos e imunológicos tem se destacado como um importante fator associado ao desenvolvimento dessa neoplasia (Pezzini e Castro, 2014).

As manifestações clínicas mais comuns são febre, perda de peso, equimoses, hepatoesplenomegalia, linfadenopatia e dores ósseo-articulares devido à infiltração medular pelos linfoblastos (Pezzini e Castro, 2014). O diagnostico se dá pela análise da medula óssea (mielograma), do sangue periférico e de testes citomorfológicos e citoquímicos, os quais permitem a classificação da LLA em L1, L2 e L3 (Callera, 2005).

O hemograma é essencial no contexto investigativo de patologias hematológicas. Recentemente, os métodos automatizados têm se mostrado extremamente uteis nas análises qualitativas e quantitativas das células sanguíneas (Pezzini e Castro, 2014). Contudo, a observação microcópica do esfregaço sanguíneo e a análise criteriosa de um médico laboratorista, bioquímico e ou biomédico é fundamental para manutenção da qualidade, exatidão e confiabilidade dos laudos emitidos (Callera, 2005; Pezzini e Castro, 2014).

A divisão do hemograma se dá pela série vermelha, série branca e série plaquetária (Pezzini e Castro, 2014).

Considerando a série vermelha, frequentemente tem se relatado anemia normocítica e normocrômica (Figura1) (Fadel, 2010). Análises mais elaboradas têm demonstrado que em $51 \%$ dos casos de LLA o nível de hemoglobina $(\mathrm{Hb})$ é menor que $7,5 \mathrm{~g} / \mathrm{dl}$, contudo, observouse também que em $80 \%$ desses casos o nível de $\mathrm{Hb}$ é inferior a $10 \mathrm{~g} / \mathrm{dl}$ (Pezzini e Castro, 2014). Portanto, a faixa de 7,5g/dl a $10 \mathrm{~g} / \mathrm{dl}$ de $\mathrm{Hb}$ pode ter um caráter sugestivo de LLA quando atrelado à clínica e a outros resultados de exames.

Figura 1. Anemia normocítica (Adaptado de Bain, 2016).

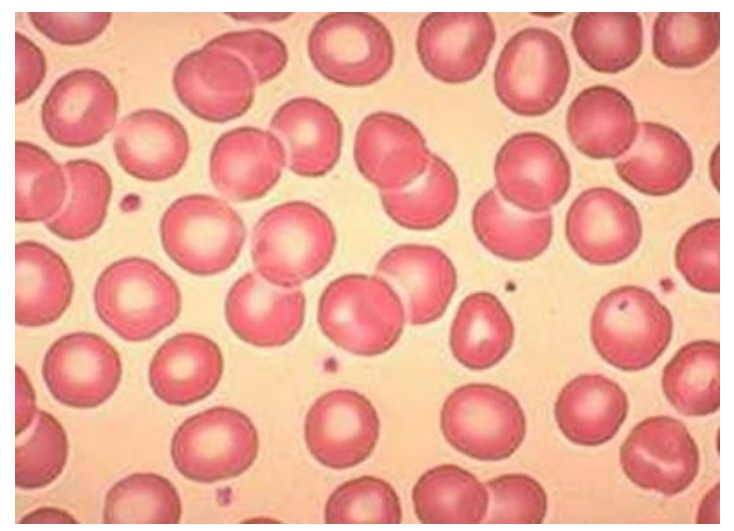


Na série branca a contagem de leucócitos pode variar da leucopenia com valores abaixo de $4000 / \mathrm{mm}^{3}$ até a leucocitose com valores maiores que $100000 / \mathrm{mm}^{3}$ (Fadel, 2010; Pezzini e Castro, 2014). Para os pacientes que apresentam leucopenia, cerca de $25 \%$ a $30 \%$ dos casos de LLA, os linfobastos são pouco frequentes no esfregaço de sangue periférico (Pezzini e Castro, 2014). Em contraste, é possível evidenciar que os linfoblastos são as células predominantes no esfregaço sanguíneo dos pacientes que apresentam leucocitose (Figura 2) (Fadel, 2010).

Figura 2. Linfoblastos que podem estar presente no sangue periférico de pacientes com LLA. (Adaptado de Bain, 2016).
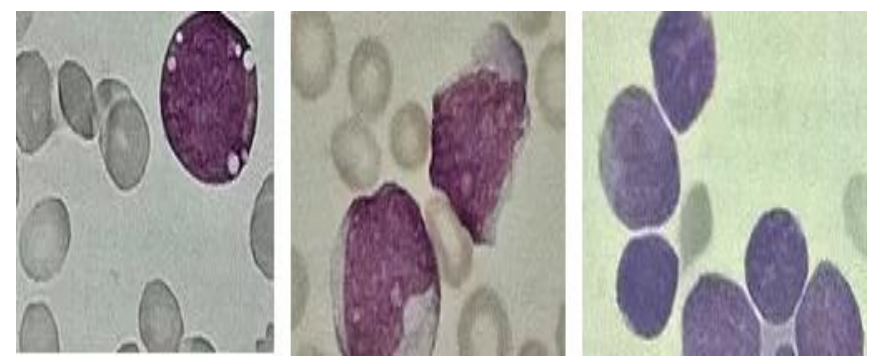

Em relação às plaquetas, trombocitopenia com valores abaixo de $150000 / \mathrm{mm}^{3}$ são comuns na LLA (FARIAS; CASTRO, 2004).

O mielograma é crucial, não só para o diagnostico da LLA, como também para sua classificação (Pezzini e Castro, 2014). Na maioria dos casos, a medula apresenta-se hipercelular com grande quantidade de linfoblastos e substituição dos espaços adiposos por precursores mielóides, eritróides residuais e megacariocitos escassos ou ausentes (Figura 3) (Fadel, 2010; Farias e Castro, 2004; Pezzini e Castro, 2014).

Figura 3. Mielograma evidenciando linfoblastos. (BARONE; FERNANDES, 2018).

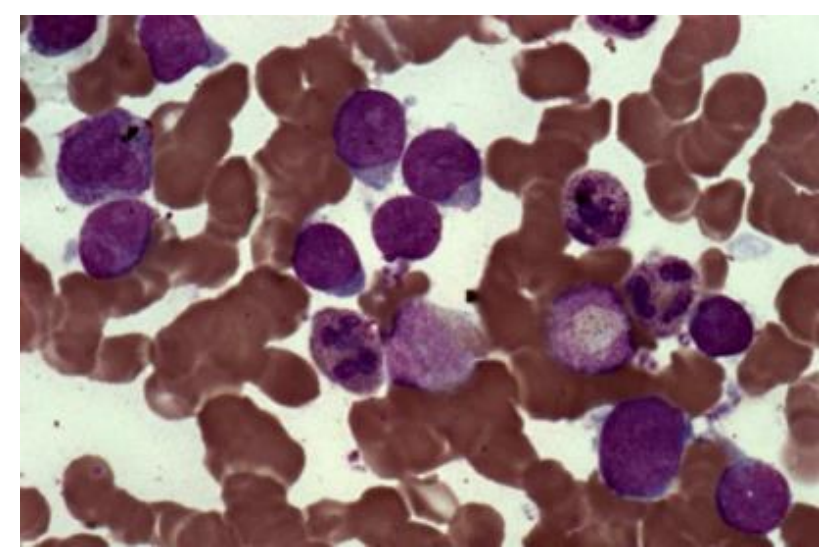

A LLA foi classificada morfologicamente pelo French American Bristh group (FAB) em três subtipos, L1, L3 e L3, baseado na morfologia do núcleo, no diâmetro celular, no número, na protuberância dos nucléolos, no volume e aspecto de e na quantidade citoplasma (Coccaro et al., 2019; Pezzini e Castro, 2014).

A LLA-L1 tem como característica a presença de células pequenas e de contorno regular, nucléolos frequentemente ausentes e citoplasma discretamente basofílico (Fadel, 2010). Na LLA-L2, tanto o tamanho das células quanto o volume do citoplasma podem variar, os nucléolos são proeminentes e irregulares e a basofilia citoplasmática é discreta (Fadel, 2010; Coccaro et al., 2019). Já na LLA-L3, as células são grandes e homogêneas com nucléolos proeminentes, vacúolos e citoplasma evidentemente basofílico (Farias e Castro, 2004). A tabela 1 e a figura 4 resumem e evidenciam as características citadas acima.

Tabela 1. Classificação morfológica da LLA (Adaptados de Farias e Castro, 2004).

\begin{tabular}{|c|c|c|c|}
\hline \multicolumn{4}{|c|}{ Classificação morfológica (FAB) da LLA } \\
\hline Morfologia & L1 & $\mathrm{L} 2$ & L3 \\
\hline $\begin{array}{l}\text { Diâmetro } \\
\text { aspecto } \\
\text { celular }\end{array}$ & $\begin{array}{l}\text { Baixo e } \\
\text { homogêneo }\end{array}$ & $\begin{array}{ll}\text { Grande } & \text { e } \\
\text { heterogênio } & \end{array}$ & $\begin{array}{l}\text { Grande } \\
\text { homogêneo }\end{array}$ \\
\hline Cromatina & $\begin{array}{l}\text { Fina ou } \\
\text { aglomerada }\end{array}$ & Fina & Fina \\
\hline $\begin{array}{l}\text { Forma do } \\
\text { núcleo }\end{array}$ & $\begin{array}{l}\text { Regular } \\
\text { com fenda } \\
\text { ou } \\
\text { indentação }\end{array}$ & $\begin{array}{lr}\text { Irregular } & \text { com } \\
\text { fenda } & \text { ou } \\
\text { indentação } & \end{array}$ & $\begin{array}{l}\text { Regular, } \\
\text { redondo ou oval }\end{array}$ \\
\hline Nucléolos & $\begin{array}{l}\text { Indistintos } \\
\text { ou não } \\
\text { visíveis }\end{array}$ & $\begin{array}{l}\text { Múltiplos } \\
\text { proeminentes }\end{array}$ & $\begin{array}{l}\text { Múltiplos } \\
\text { proeminentes }\end{array}$ \\
\hline $\begin{array}{l}\text { Volume } \\
\text { citoplasmático }\end{array}$ & Escasso & $\begin{array}{l}\text { Moderadamente } \\
\text { abundante }\end{array}$ & $\begin{array}{l}\text { Moderadamente } \\
\text { abundante }\end{array}$ \\
\hline $\begin{array}{l}\text { Basofilia } \\
\text { citoplasmática }\end{array}$ & Ligeira & Ligeira & Evidente \\
\hline $\begin{array}{l}\text { Vacúolos } \\
\text { citoplasmático }\end{array}$ & Variável & Variável & Evidentes \\
\hline
\end{tabular}


Figura 4. Características morfológicas da LLA-L1, LLAL2 e LLA-L3. (Barone e Fernandes, 2018).
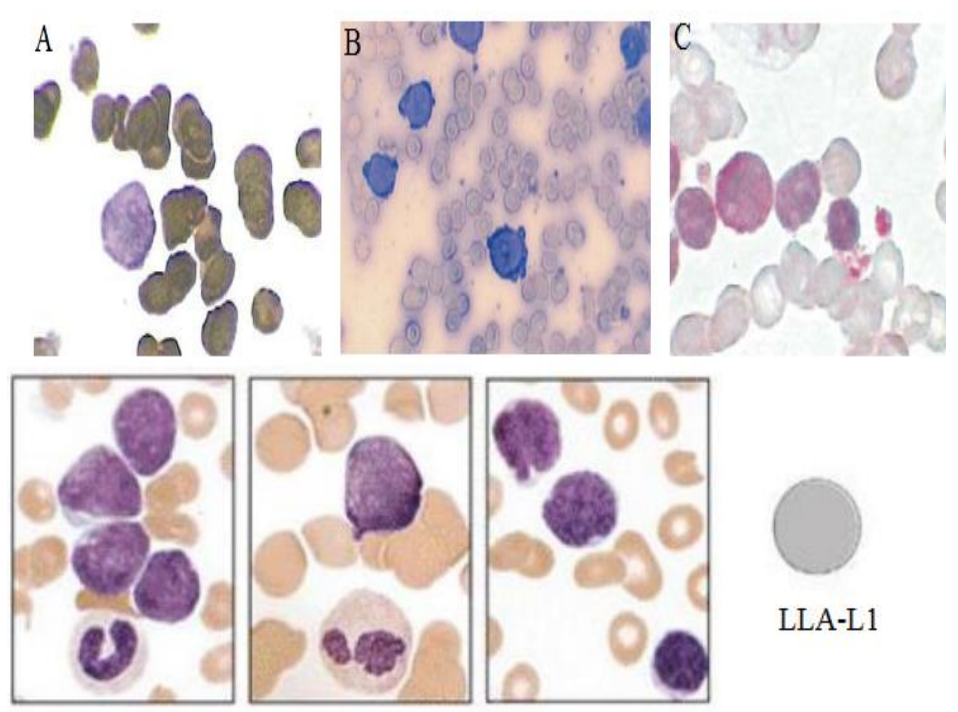

LLA-L1
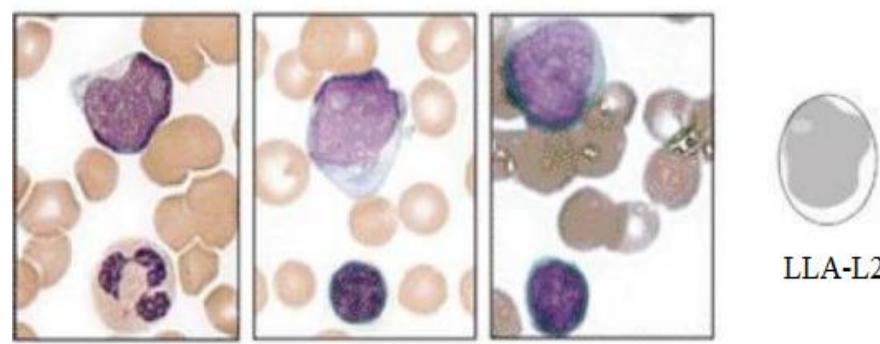

LLA-L2
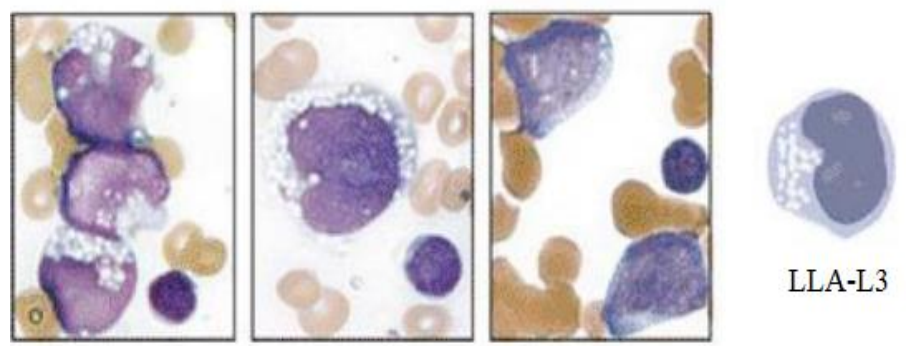

LLA-L3

Análises cito químicas auxiliam na diferenciação entre LLA e LMA (Farias e Castro, 2004). As reações de mieloperoxidase e sudan black mostram-se uniformemente negativas para os linfoblastos, o que não acontece para os mieloblastos (Figura 5) (Fadel, 2010; Fujita et al., 2021). Na coloração de ácido periódico de Schiff, embora exista negatividade para a linhagem $\mathrm{T}$, os linfoblastos geralmente são positivos e exibem uma coloração em forma de anéis concêntricos de grânulos grosseiros ou em blocos maciços, já os mieloblastos, quando positivos não exibem tal aspecto granular (Figura 5) (Callera, 2005; Fujita et al., 2021; Pezzini e Castro, 2014)
Figura 5. Citoquímica da LLA. (A) Mieloperoxidase, (B) Sudan black, (C) coloração de ácido periódico de Schiff. (Adaptado de Callera, 2005).
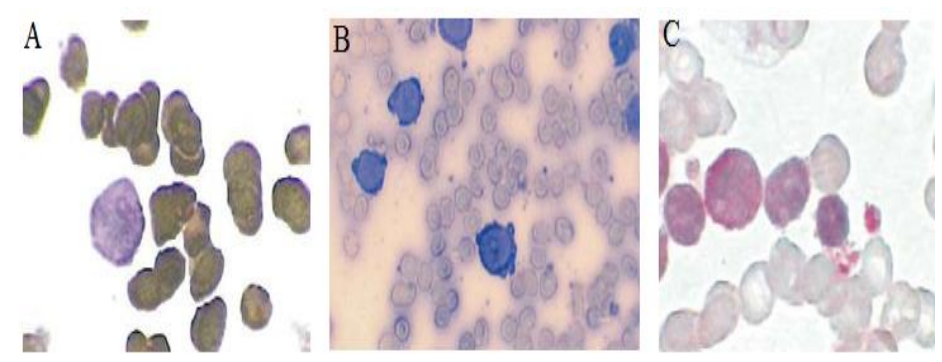

A imunofenotipagem é fundamental para o diagnostico, determinação da linhagem dos linfoblastos e prognóstico da LLA (Pezzini e Castro, 2014). Inúmeros autores caracterizam a LLA em linhagem B ou T de acordo com a expressão de antígenos específicos denominados cluster designations (CDs) (Inaba e Mullighan, 2020; Pezzini e Castro, 2014). A técnica de citometria de fluxo é rápida e objetiva, o que permite determinar a linhagem dos linfoblastos, os quais devem expressar no mínimo dois antígenos específicos para a uma dada linhagem, como por exemplo, CD79a e CD19cito para linhagem B e CD7+ e CD3+ para linhagem T (Callera, 2005; Fadel, 2010; Pezzini e Castro, 2014). A tabela 2 mostra o perfil imunofenotípico e os CDs expressos pelas linhagens T e B da LLA (Inaba e Mullighan, 2020; Malard e Mohty, 2020).

A LLA de linhagem B foi estratificada de acordo com os estágios de diferenciação dos progenitores $\mathrm{B}$ na medula, sendo divididas em LLA pró-B, comum, pré-B e Bmadura (Farias e Castro, 2004). A LLA do tipo pró-B representa 5\% dos casos em crianças e $10 \%$ em adultos (Callera, 2005; Fadel, 2010). A LLA do tipo comum representa $75 \%$ dos casos em crianças e $50 \%$ em adultos (Pezzini e Castro, 2014). A leucemia pré-B representa 15\% dos casos em crianças com LLA e 10\% em adultos (Fadel, 2010). Já a LLA do tipo B maduro está presente em 2\% a 5\% em crianças e adultos (Farias e Castro, 2004). Esta ultima, apresentam blastos com características morfológicas L3 e é a leucemia que apresenta o pior prognostico, pois compromete o sistema nervoso central e a resposta à terapia geralmente é refratária (Coccaro et al., 2019; Fujita et al., 2021).

A LLA de linhagem T é dividida em LLA pré-T, Tintermediária e T-madura e acomete cerca de $25 \%$ dos adultos e $15 \%$ das crianças, além disso, indivíduos do sexo masculino se mostram mais propensos a desenvolver a doença (Callera, 2005; Fujita et al., 2021). 
Tabela 2. Perfil imunofenotípico das linhagens da LLA (Adaptado de Pezzini e Castro, 2014).

\begin{tabular}{|c|c|c|c|c|c|c|c|}
\hline \multicolumn{8}{|c|}{ Imunofenotipagem das linhagens da LLA } \\
\hline \multirow[b]{2}{*}{ Marcador } & \multicolumn{3}{|c|}{ Linhagem B } & \multirow[b]{2}{*}{ B } & \multicolumn{2}{|c|}{$\begin{array}{c}\text { Linhagem } \\
T\end{array}$} & \\
\hline & $\begin{array}{l}\text { Pró } \\
-B\end{array}$ & $\begin{array}{c}\mathrm{C} \\
\mathrm{o} \\
\mathrm{m} \\
\mathrm{u} \\
\mathrm{m}\end{array}$ & $\begin{array}{l}\text { Pré } \\
-B\end{array}$ & & $\begin{array}{l}\text { Pré- } \\
\mathrm{T}\end{array}$ & $\begin{array}{l}\text { Int } \\
\text { er } \\
\text { me } \\
\text { diá } \\
\text { rio }\end{array}$ & $\mathrm{T}$ \\
\hline HLA-DR & + & + & + & + & $+/-$ & - & - \\
\hline TdT & + & + & + & + & + & + & + \\
\hline CD19 & + & + & + & + & - & - & - \\
\hline $\mathrm{CD} 22 \mathrm{c}$ & $-/+$ & + & + & + & - & - & - \\
\hline CD10 & - & + & + & 1+ & $-/+$ & $-/+$ & $\overline{-}$ \\
\hline CD20 & - & $-/+$ & + & + & - & - & - \\
\hline$c \mu$ & - & - & + & - & - & - & - \\
\hline SmIg & - & - & - & + & - & - & - \\
\hline CD7 & - & - & - & - & + & + & + \\
\hline $\mathrm{CD} 2$ & - & - & - & - & - & + & + \\
\hline CD3(c) & - & - & - & - & $-/+$ & + & + \\
\hline CD1a & - & - & - & - & - & $-/+$ & - \\
\hline CD3 & & - & - & - & - & - & + \\
\hline $\begin{array}{c}\mathrm{CD} 4 / \mathrm{CD} \\
8\end{array}$ & - & - & - & - & - & $-/+$ & - \\
\hline
\end{tabular}

\section{Conclusão}

A LLA é a neoplasia hematológica mais comum em crianças, contudo, pode acometer uma porcentagem significativa da população adulta. Caracterizada pelo início súbito, rápida evolução e pela presença maciça de linfoblastos na medula óssea e em outros órgãos, seu reconhecimento e diagnostico é vital estabelecer o prognostico clínico e dirigir a melhor conduta terapêutica para um dado caso.

Embora o hemograma não defina um diagnostico definitivo para a LLA, achados como a anemia normocítica normocrômica, a trombocitopenia e a presença de linfoblastos podem fornecer indícios essenciais de um possível caso e justificar testes e análises confirmatórias como o mielograma, citoquímica e imunofenotipagem.

Nesse contexto, portanto, o profissional do laboratório de hematologia, seja ele, médico laboratorista, bioquímico e ou biomédico, deve estar apto e atento na identificação das alterações que podem sugerir e até confirmar, dependendo da estrutura do laboratório, a suspeita de um caso de LLA.

\section{Declaração} interesse.

Os autores declaram que não existe conflito de

\section{Referências}

Brasil. Ministério da Saúde. Estimativa de 2018: Incidência de câncer no Brasil. Disponível em https://www.inca.gov.br/sites/ufu.sti.inca.local/files//me dia/document//estimativa-incidencia-de-cancer-nobrasil-2018.pdf. Acesso em: Out. 2019.

Bain BJ. Células sanguíneas - Um guia prático $5^{\circ}$ edição. Porto Alegre: Artmed, 2016.

Baroni A, Fernandes A. Leucemias. Disponível em: http://www.profbio.com.br/aulas/hemato1_10.pdf. Acesso em: 21 Jul. 2018.

Callera F. Bilineal acute leukemia: a rare expression of common all markers and pre-T markers. Revista Brasileira de Hematologia e Hemoterapia, São José do Rio Preto, 27, 70-71, 2005.

Coccaro N, Anelli L, Zagaria A, Specchia G, Albano F. NextGeneration Sequencing in Acute Lymphoblastic Leukemia. International Journal of Molecular Sciences, $15,20,1-22,2019$

Fadel, A.P. Investigação laboratorial de Leucemia Linfóide Aguda. $A C \& T$ CIENTÍFICA, São José do Rio Preto, 1, 1-10, 2010.

Farias MG, Castro SM. Diagnóstico laboratorial das leucemias linfóides agudas. Jornal Brasileiro de Patologia $e$ Medicina Laboratorial, 40, 2, 91-98, 2004.

Fujita TC, Sousa-Pereira N, Amarante MK, Watanabe MAE. Acute lymphoid leukemia etiopathogenesis. Molecular Biology Reports, 48, 1, 817-822, 2021.

Inaba H, Mullighan CG. Pediatric acute lymphoblastic leukemia. Haematologica, 105, 11, 2524-2539, 2020.

Malard F, Mohty M. Acute lymphoblastic leukaemia. Lancet, 4, 395, 1146-1162, 2020.

Pezzini TJ, Castro FS. Alterações hematológicas na leucemia Linfóide aguda (LLA). Estudos, 41, 4, 767-776, 2014. 